\title{
Short- and Long-Term Plasticity at the Axon Initial Segment
}

\author{
Matthew S. Grubb, ${ }^{1}$ Yousheng Shu, ${ }^{2}$ Hiroshi Kuba, ${ }^{3}$ Matthew N. Rasband, ${ }^{4}$ Verena C. Wimmer, ${ }^{5}$ and Kevin J. Bender ${ }^{6}$ \\ ${ }^{1}$ MRC Centre for Developmental Neurobiology, King's College London, London SE1 1UL, United Kingdom, ${ }^{2}$ Institute of Neuroscience and State Key \\ Laboratory of Neuroscience, Shanghai Institutes for Biological Sciences, Chinese Academy of Sciences, Shanghai 200031, China, ${ }^{3}$ Department of Physiology, \\ Kyoto University Graduate School of Medicine, Kyoto 606-8501, Japan, ${ }^{4}$ Department of Neuroscience, Baylor College of Medicine, Houston, Texas 77030, \\ ${ }^{5}$ Florey Neuroscience Institutes, The University of Melbourne, Parkville 3010, Victoria, Australia, and ${ }^{6}$ Gallo Research Center and Department of Neurology, \\ University of California, San Francisco, Emeryville, California 94608
}

The axon initial segment (AIS) is a highly specialized neuronal subregion that is the site of action potential initiation and the boundary between axonal and somatodendritic compartments. In recent years, our understanding of the molecular structure of the AIS, its maturation, and its multiple fundamental roles in neuronal function has seen major advances. We are beginning to appreciate that the AIS is dynamically regulated, both over short timescales via adaptations in ion channel function, and long timescales via activitydependent structural reorganization. Here, we review results from this emerging field highlighting how structural and functional plasticity relate to the development of the initial segment, and to neuronal disorders linked to AIS dysfunction.

\section{Introduction}

The majority of neurons are polarized into dendrites that receive synaptic input and axons that transmit action potential output. The length of the axon immediately distal to the axon hillock, termed the axon initial segment (AIS), separates these two regions and serves multiple neuronal functions. Electrically, the AIS bridges dendritic and axonal compartments, converting graded dendritic inputs into all-or-none action potentials. Molecularly, the AIS maintains neuronal polarity by filtering for axon-bound cellular cargo. To perform these tasks, the AIS is imbued with a rich array of ion channels, associated anchoring proteins, and cytoskeletal components (Fig. 1). While the basic building blocks of the AIS have been known for over a decade, recent studies have revealed an ever-expanding complexity in the molecular components, and in the types and distribution of ion channels enriched in the AIS.

This complexity underlies what is now being recognized as a highly dynamic structure. As highlighted in this review, the AIS is not merely a trigger zone for action potentials, but rather is a site for complex neuronal processing. Initial segment structure and composition varies considerably across, and even within, neuronal classes, seemingly tuned to the computational demands of the cell. A cell's recent activity can affect AIS ion channel kinetics and availability, thus altering action potential waveform, timing, and probability. In some cells, the AIS is a direct target for ionotropic

Received Aug. 8, 2011; revised Sept. 9, 2011; accepted Sept. 9, 2011.

This work was supported by a Wellcome Trust Career Development Fellowship (M.S.G.), 973 Program Grants 2011CBA00400 and 2006CB806600 and the National Natural Science Foundation of China Project Grant 31025012 (Y.S.), Grants-in-Aid from Ministry of Education, Culture, Sports, Science, and Technology (22680032) and the Japan Science and Technology Agency Precursory Research for Embryonic Science and Technology program (H.K.), NIH Grants NS044916 and NS069688 (M.N.R.), and NIH Grant DC011080 and funds from the state of California for medical research on alcohol and substance abuse through the University of California, San Francisco (K.J.B.).

Correspondence should be addressed to either Matthew $S$. Grubb or Kevin J. Bender at the above addresses. E-mail: matthew.grubb@kcl.ac.uk or kbender@gallo.ucsf.edu.

DOI:10.1523/JNEUROSCI.4064-11.2011

Copyright $\odot 2011$ the authors $\quad 0270-6474 / 11 / 3116049-07 \$ 15.00 / 0$ and metabotropic transmission, which in turn dramatically alters spike output. Over long timescales, even the location and size of the AIS can change to compensate for alterations in neuronal activity.

These new findings are forcing a shift in our understanding of neuronal plasticity in health and disease, as the AIS must now be considered a site for structural and functional change within neurons. Here, we detail the mechanisms and implications of these various forms of structural and functional plasticity found at the AIS, the development and regulation of this structure, and how these processes relate to disease.

\section{AIS ion channel function and modulation}

The AIS is enriched with multiple types of ion channels, including $\mathrm{Na}^{+}, \mathrm{K}^{+}$, and-more recently identified- $\mathrm{Ca}^{2+}$ channels (Fig. 1). These channels play distinct roles in action potential initiation, and variations in the complement of ion channels expressed in a given cell contribute to the rich diversity in action potential waveforms observed across neuronal classes (Bean, 2007). The biophysical characteristics of these channels not only determine a neuron's fundamental spike waveform, but also how firing patterns change in response to recent activity.

Although multiple neuronal sites can support action potential generation, the high density of $\mathrm{Na}^{+}$channels inherent to the AIS makes it the lowest threshold site for initiation. Immunostaining, imaging of spike-dependent $\mathrm{Na}^{+}$flux, and electrophysiological studies suggest a similar density of $\mathrm{Na}^{+}$channels throughout the AIS (Colbert and Pan, 2002; Kole et al., 2008; Lorincz and Nusser, 2008; Bender and Trussell, 2009; Fleidervish et al., 2010); however, careful analysis of action potential initiation and propagation, including the use of newly developed voltage imaging techniques, show that they preferentially initiate at the distal end of the AIS (Palmer and Stuart, 2006; Hu et al., 2009; Popovic et al., 2011). What makes the distal end of the AIS privileged for spike initiation? Equipped with a novel method of axon-bleb patch-clamp recording (Shu et al., 2006, 2007a,b), together with 
somatic nucleated patch recording from neocortical pyramidal cells, a recent study revealed that $\mathrm{Na}^{+}$channels at the distal AIS and the adjacent axon have a much lower half-activation voltage (up to 14 $\mathrm{mV}$ ) than those at the proximal AIS and the soma (Hu et al., 2009). Consistent with these electrophysiological data, immunostaining results revealed a segregation of $\mathrm{Na}^{+}$channel subtypes at the AIS, with high-threshold $\mathrm{Na}_{\mathrm{V}} 1.2$ channels and low-threshold $\mathrm{Na}_{\mathrm{V}} 1.6$ channels targeted preferentially to the proximal and the distal AIS, respectively (Fig. $1 A, D$ ) (Hu et al., 2009). Immunostaining intensity of $\mathrm{Na}_{\mathrm{V}} 1.6$ peaked at the distal end of the AIS, corresponding well to the action potential initiation zone. A biophysical model indicated that the voltage threshold for action potential initiation was largely determined by the density of $\mathrm{Na}_{\mathrm{V}} 1.6$, whereas $\mathrm{Na}_{\mathrm{V}} 1.2$ channels in the proximal AIS support action potential backpropagation into the somatodendritic compartment (Fig. 2A). Indeed, when somatic membrane potential is hyperpolarized, axonal action potentials can fail to backpropagate (Hu et al., 2009). Therefore, AIS $\mathrm{Na}_{\mathrm{V}} 1.6$ and $\mathrm{Na}_{\mathrm{V}} 1.2$ channels may make distinct contributions to orthodromic action potential initiation and antidromic backpropagation. It will be of great interest to determine whether these $\mathrm{Na}_{\mathrm{V}}$ isoforms are subject to plasticity mechanisms that could differentially affect orthodromic and antidromic spike generation.

Initial segment $\mathrm{K}^{+}$channels contribute to both resting membrane potential and action potential repolarization. Recent studies have found that $\mathrm{K}_{\mathrm{V}} 1$ channels are distributed in the AIS in multiple cell types (Fig. 1D) (Kole et al., 2007; Shu et al., 2007a; Goldberg et al., 2008). These channels activate rapidly but inactivate very slowly in response to modest somatic depolarizations. Since $\mathrm{K}_{\mathrm{V}} 1$ channel expression is restricted to the axon, their inactivation has little effect on backpropagating action potentials, but does alter orthodromic axonal action potentials by increasing their half-width (Fig. $2 B$ ). This in turn facilitates downstream neurotransmitter release, altering the output of downstream neurons (Kole et al., 2007; Shu et al., 2007a; Zhu et al., 2011).

Fluorescence imaging techniques have revealed spike-evoked $\mathrm{Ca}^{2+}$ influx in the AIS of cortical layer $5 \mathrm{~b}$ pyramidal neurons, cerebellar Purkinje neurons, dorsal root ganglion neurons, and brainstem interneurons (Schiller et al., 1995; Callewaert et al., 1996; Lüscher et al., 1996; Bender and Trussell, 2009; Yu et al., 2010). The source of this $\mathrm{Ca}^{2+}$ influx has been most thoroughly investigated in cartwheel cells, which are glycinergic interneurons found in the auditory brainstem. These cells fire spike bursts typified by the recruitment of subthreshold $\mathrm{Ca}^{2+}$ influx through low voltage-activated (LVA) $\mathrm{Ca}^{2+}$ channels, which include Tand R-type isoforms (Perez-Reyes, 2003). Traditionally, burstevoked $\mathrm{Ca}^{2+}$ influx was thought to be restricted to the dendrites, but, interestingly, $\mathrm{Ca}^{2+}$ influx in the AIS was mediated by these same LVA channels (Fig. 2C) (Bender and Trussell, 2009). Their localization to the AIS, and the unique biophysical properties of LVA channels, suggests that these channels heavily influence spike initiation. Since LVA channels activate at hyperpolarized potentials, they contribute to subthreshold depolarization of the AIS. Thus, their activity can determine when and if a given stimulus evokes an action potential. Compared to $\mathrm{Na}^{+}$or $\mathrm{K}^{+}$channels, LVA $\mathrm{Ca}^{2+}$ channels activate and inactivate over much longer timescales, and therefore play a critical role in the generation of spike bursts. Indeed, a partial block of AIS Ca ${ }^{2+}$ influx is all that is needed to convert what would be a spike burst into a single spike (Bender and Trussell, 2009).

While short-term, activity-dependent changes in ion channel function, including changes in activation and inactivation, have been observed for both AIS $\mathrm{Na}^{+}$and $\mathrm{K}^{+}$channels (see above), there has never been direct evidence for neuromodulation of AIS ion channels. $\mathrm{Ca}^{2+}$ channels, in particular T-type channels, are common targets for neuromodulation, and indeed, a recent study showed that $\mathrm{Ca}^{2+}$ influx through cartwheel cell AIS T-type channels was regulated by a type 3 dopamine receptor $\left(D_{3} R\right)$-dependent pathway (Bender et al., 2010). This neuromodulatory pathway was remarkably specific for just AIS T-type channels; neither dendritic T-type channels nor AIS $\mathrm{Na}^{+}$or $\mathrm{K}^{+}$channels were affected by $\mathrm{D}_{3} \mathrm{R}$ signaling. Dopamine receptor activation produced relatively modest reductions in overall AIS $\mathrm{Ca}^{2+}$ influx, but consistent with partial block of AIS $\mathrm{Ca}^{2+}$ with channel antagonists, these small changes in $\mathrm{Ca}^{2+}$ flux had dramatic effects on burst output, reducing the number of spikes per burst by $50 \%$ (Fig. 2 C) (Bender and Trussell, 2009).

Our understanding of AIS ion channels has been limited by our ability to directly access this small neuronal compartment. This barrier is being overcome through a variety of techniques described above, including direct whole-cell recording, imaging of local ion flux, and imaging of membrane voltage dynamics. These technical breakthroughs have revealed a surprising level of plasticity in AIS ion channel function in response to recent activity and also to neuromodulators. Equipped with these new tools, future studies will likely show that these plasticity mechanisms are just the first of many ways in which neuronal function is controlled at the initial segment. 
A

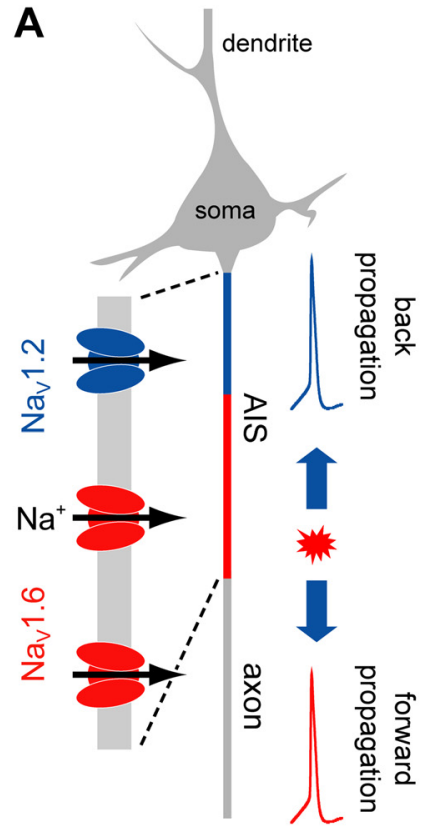

B
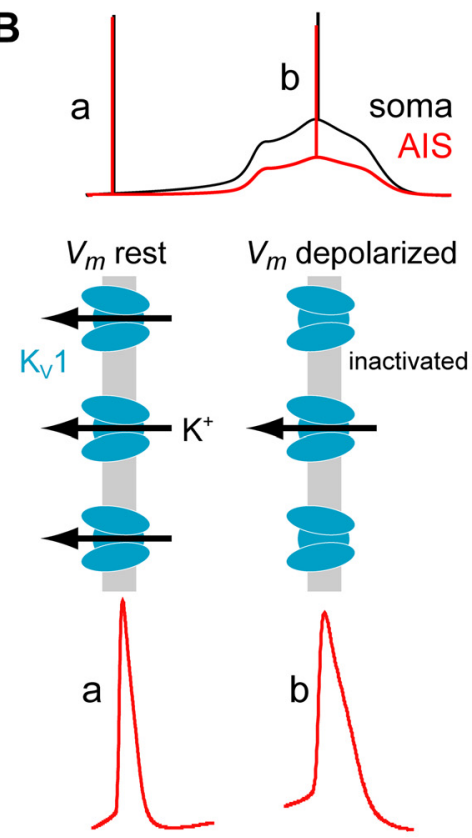
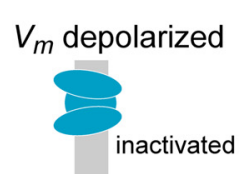

inactivated

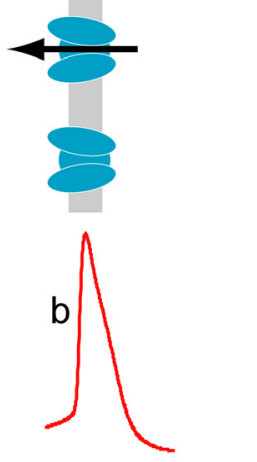

C

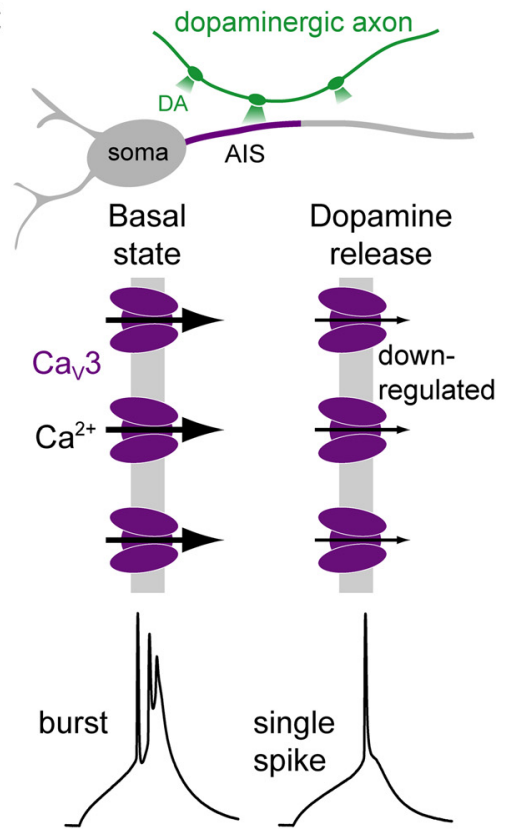

Figure 2. Function and modulation of AIS ion channels. A, Schematic detailing roles of $\mathrm{Na}^{+}$channel subtypes in AP initiation and backpropagation in neocortical pyramidal neurons. Accumulation of the low-threshold $\mathrm{Na}_{\mathrm{v}} 1.6$ channels at the distal AIS determines the lowest threshold for AP initiation, whereas accumulation of the high-threshold Na 1.2 channels at the proximal AIS promotes AP backpropagation to the soma and dendrites. $\boldsymbol{B}$, Axonal $\mathrm{K}_{\mathrm{V}} 1$ channels can shape the waveforms of axonal APs. Spreading of the somatic subthreshold depolarizations into the axon (top) may inactivate some axonal $K_{v} 1$ channels (middle), increasing axonal AP duration (bottom). This in turn enhances neurotransmitter release. C, AlS low-voltage activated Ca ${ }^{2+}$ channels (Ca 3 ) promote AP bursts in auditory brainstem interneurons. Their downregulation via local dopaminergic signaling suppresses spike output in response to somatic depolarization. DA, Dopamine.

\section{Long-term activity-dependent plasticity of AIS position and length}

While the initial segment's constituent ion channels are subject to significant short-term plasticity, recent studies have also revealed that on longer timescales the AIS is more diverse and dynamic than previously thought. Ion channel composition at the AIS can vary considerably across different neuronal types (Lorincz and Nusser, 2008), and diversity in AIS length and axonal position may also underlie interneuronal variation in firing properties. For example, classes of retinal ganglion cells with different visual properties have initial segments that differ in length, and in their position within the axon (Fried et al., 2009). In the avian nucleus magnocellularis $(\mathrm{NM})$ and nucleus laminaris $(\mathrm{NL})$, which are the second- and third-order nuclei in the auditory pathway (Konishi, 2003), the length and the location of the AIS varies with the tuning frequency of neurons. Initial segments are shorter in neurons with higher tuning frequency in the NM, and are shorter and more distally localized in neurons with higher tuning frequency in NL (Kuba et al., 2006; Kuba and Ohmori, 2009). Computational models show that this AIS diversity is optimally tuned to each neuron's synaptic inputs, thus playing a critical role in optimizing auditory signal processing in these nuclei (Kuba et al., 2006). Although AIS variation can be crucial for neuronal function, until recently the mechanisms controlling AIS location and length remained entirely unknown. Two studies published last year have revealed a crucial regulatory element determining AIS position and size both in vitro and in vivo: electrical activity.

First, chronically increasing neuronal activity over a $48 \mathrm{~h}$ period in cultured hippocampal neurons resulted in a significant shift in AIS position, with the entire structure moving distally along the axon away from the soma (Fig. 3) (Grubb and Burrone, 2010). This distal shift occurred whether activity was increased globally via elevated extracellular potassium, or on a tightly spatiotemporally controlled basis using the light-activated ion chan- nel channelrhodopsin-2 (ChR2). It involved all AIS components tested, including vital scaffolding molecules such as ankyrin $\mathrm{G}$ (ankG) and $\beta \mathrm{IV}$-spectrin, as well as the $\mathrm{Na}^{+}$channels underlying action potential (AP) initiation. In addition, recent experiments have shown that the distribution of ion channel subunits within the AIS (Hu et al., 2009) (see above, Fig. 1D) is also maintained after activity-dependent relocation: $\mathrm{Na}_{\mathrm{v}} 1.6, \mathrm{~K}_{\mathrm{v}} 1.1$, and $\mathrm{K}_{\mathrm{v}} 1.2$ channel subunits all remain preferentially localized in the distal AIS following $48 \mathrm{~h}$ high potassium treatment (Evans and Grubb, 2011). Importantly, hippocampal AIS relocation is a bidirectional phenomenon-as well as moving away from the soma in response to increased activity, the AIS can shift proximally after neurons are returned to baseline activity conditions. In this way, ongoing neuronal activity can fine-tune AIS position.

What activity-dependent signals do neurons detect to trigger AIS relocation? The available evidence points heavily at increases in intracellular calcium. Both high $\mathrm{K}^{+}$treatment and successful ChR2-based activity patterns were characterized by large and sustained elevations in internal $\left[\mathrm{Ca}^{2+}\right]$, and while blocking APs did not prevent AIS relocation, blocking L-type $\mathrm{Ca}^{2+}$ channels did (Grubb and Burrone, 2010). Although there are specialized internal calcium stores at the AIS (Sánchez-Ponce et al., 2011) and AIS-based $\mathrm{Ca}^{2+}$ channels that can shape APs, local $\mathrm{Ca}^{2+}$ signals seem unlikely to trigger long-term AIS plasticity. L-type $\mathrm{Ca}^{2+}$ channels have not yet been functionally detected at the AIS (Bender and Trussell, 2009; Yu et al., 2010), and pharmacological manipulations of $\mathrm{Ca}^{2+}$-dependent $\mathrm{Ca}^{2+}$ release do not prevent the depolarization-induced distal shift in AIS location (Evans and Grubb, 2011). Instead, somatic detection of cell-wide activity changes may underlie this form of neuronal plasticity.

The second recent study of activity-dependent AIS plasticity was motivated by the optimal frequency tuning produced by AIS properties in the chick auditory brainstem (Kuba et al., 2006). Could these AIS properties be modulated by presynaptic activity 
in vivo? This was indeed found in a study where chicks were monaurally deprived by unilateral cochlea removal and the effects examined in the NM (Kuba et al., 2010). Within $7 \mathrm{~d}$ after deprivation of afferent inputs, the distribution of $\mathrm{Na}^{+}$ channels at the AIS elongated by $>50 \%$ in the deprived NM, with little change in their density and composition (Fig. 3). The expansion of $\mathrm{Na}^{+}$channel territory was accompanied by changes in ankG distribution, indicating that the elongation reflects a true change in AIS structure. Elongation of the AIS also occurred with less severe manipulations, such as tympanic membrane puncture or immobilization of middle ear bones, which attenuate auditory inputs without damaging cochlea hair cells (Tucci and Rubel, 1985). This confirms that a decrease in presynaptic activity, rather than cochlear damage per se, triggers longterm AIS plasticity.

These two papers show that electrical activity can shape fundamental properties of the AIS, but while a lack of auditory input causes a change in AIS length (Kuba et al., 2010), increased levels of hippocampal neuron activity produce a shift in AIS location (Grubb and Burrone, 2010). Why the differing effects? Interspecies, developmental, or methodological differences between the two studies could always be responsible; however, distinctions in neuronal type may be a more likely explanation. NM neurons are highly specialized adendritic cells with short initial segments $(\sim 10 \mu \mathrm{m})$ under normal conditions, so it may be no surprise that their response to electrical activity perturbation is different from that of hippocampal neurons, whose initial segments are usually much longer $(\sim 30 \mu \mathrm{m})$. Indeed, cell type-based variability in AIS plasticity is seen within hippocampal cultures, where GAD65expressing interneurons display little or no shift in AIS location upon high potassium stimulation (Grubb and Burrone, 2010). More surprisingly, dopaminergic neurons in dissociated cultures of rat olfactory bulb show inverse AIS plasticity: their initial segments move proximally after $48 \mathrm{~h}$ depolarization (Chand and Grubb, 2011). It will be fascinating in the future to see how, and why, different types of neuron from diverse brain regions use different forms of AIS plasticity in response to perturbations in their electrical activity.

Finally, how might neurons benefit from activity-dependent long-term AIS plasticity? Given that the AIS is the site of AP initiation, altering its structural properties should be an efficient mechanism for controlling neuronal activity. Indeed, both recent studies show that AIS plasticity is coupled with changes in neuronal excitability: the longer AISs produced by auditory deprivation resulted in higher axonal $\mathrm{Na}^{+}$current density and lower spike threshold in NM neurons (Kuba et al., 2010), while the more distal AISs in overactive hippocampal neurons were associated with decreased neuronal excitability (Fig. 3) (Grubb and Burrone, 2010). AIS plasticity may therefore work as a homeostatic mechanism, operating in concert with other well defined processes of synaptic and intrinsic excitability homeostasis to either increase activity after the silencing of synaptic input, or decrease excitability after neuronal overstimulation (Marder and Prinz, 2002; Turrigiano and Nelson, 2004). Notably, cochlear deprivation disrupted the tuning frequency dependence of AIS length in the NM (Kuba et al., 2010), suggesting that AIS-based, homeostatic fine-tuning of neuronal activity could be especially crucial for optimizing neuronal circuitry in the developing brain.

\section{AIS assembly and maintenance}

The results described above emphasize the importance of ion channels at the AIS, and how their location, biophysical properties, and distribution can be modified by activity. However, how activity directly regulates these AIS properties, and whether the phenomenon of AIS plasticity occurs during normal brain function or only in response to large perturbations in neuronal activity or pathological conditions remains unclear. One strategy to begin to elucidate the mechanisms regulating AIS plasticity is to determine how the AIS is assembled during development and then maintained over an organism's lifetime.

How do ion channels become enriched at the AIS? As described above, the AIS is highly enriched in a variety of ion channels and each interacts with scaffolding proteins that link to the flexible actin/ $\beta$ IV spectrin-based submembranous cytoskeleton (Fig. 1D). Two scaffolding proteins have been identified at the AIS: ankG and PSD-93. PSD-93 binds to the $\mathrm{K}_{\mathrm{V}} 1$ channels found at the AIS, and silencing of PSD-93 expression in cultured hippocampal neurons blocked the recruitment of $\mathrm{K}_{\mathrm{V}} 1$ channels to the AIS (Ogawa et al., 2008). However, loss of PSD-93 did not affect the clustering of $\mathrm{Na}^{+}$channels or other AIS proteins. Furthermore, accumulation of $\mathrm{K}_{\mathrm{V}} 1$ channels occurs after the clustering of $\mathrm{Na}^{+}$and $\mathrm{KCNQ} 2 / 3 \mathrm{~K}^{+}$channels, suggesting that the mechanisms responsible for $\mathrm{Na}^{+}$channel localization to the AIS are upstream of $\mathrm{K}_{\mathrm{V}} 1$ channel clustering and may be organizers of AIS assembly and function. Both $\mathrm{Na}^{+}$and $\mathrm{KCNQ} 2 / 3 \mathrm{~K}^{+}$channels have similar AIS targeting motifs that interact with ankG to mediate channel localization (Garrido et al., 2003; Lemaillet et al., 2003; Hill et al., 2008). Indeed, ablation of ankG expression either through shRNA or genetic methods blocked the clustering of $\mathrm{Na}^{+}$ and $\mathrm{K}^{+}$channels (Zhou et al., 1998; Pan et al., 2006; Hedstrom et al., 2007). In addition to ion channels, the AIS is also enriched in the cell adhesion molecules NrCAM and Neurofascin-186, and the cytoskeletal linker $\beta$ IV spectrin (Fig. $1 D$ ). Recruitment of these proteins to the AIS also depends on ankG (Davis and Bennett, 1994; Yang et al., 2007). Together, these results point to ankG as the master regulator of AIS assembly.

How are ion channels maintained and restricted to the AIS for long periods of time? This question is important since the forms 
of AIS plasticity described above take place over the course of hours and days, rather than the timescale of minutes. Silencing of AIS proteins in mature, fully polarized neurons in culture revealed that as for development, ankG is required to maintain ion channels at the AIS (Hedstrom et al., 2008). Intriguingly, these and other experiments also showed that ankG functions not only to cluster and maintain ion channels, but also to maintain neuronal polarity, i.e., the distinction between axonal and somatodendritic domains (Hedstrom et al., 2008; Sobotzik et al., 2009). As there are many signaling pathways that regulate axon-dendrite polarity (Barnes and Polleux, 2009), it will be interesting to determine whether any contribute to neuronal polarity by regulating the position of ankG along the axon.

The importance of ankG, and the associated $\beta$ IV spectrin, in maintenance of neuronal polarity and ion channel clustering is emphasized by the observation that under conditions of ischemic brain injury both ankG and $\beta \mathrm{IV}$ spectrin are rapidly proteolyzed by the calcium-dependent protease calpain, and this can occur independently of cell death or axon degeneration (Schafer et al., 2009). Proteolysis of ankG and $\beta$ IV spectrin resulted in loss of ion channels and loss of neuronal polarity. It will be interesting to determine whether the dismantling of the AIS as seen during stroke and other injuries are extreme examples of plasticity gone awry. Thus, discerning mechanisms of injury that also affect AIS structure and function may lead to insights into AIS plasticity that do not involve total destruction of the AIS.

How might interactions with ankG be regulated to facilitate AIS plasticity? A particularly intriguing example is the differential localization of $\mathrm{Na}_{\mathrm{V}} 1.2$ and $\mathrm{Na}_{\mathrm{V}} 1.6$ to proximal and distal regions of the AIS (Fig. 1A,D) (Van Wart and Matthews, 2006). Since both of these $\mathrm{Na}^{+}$channels share the common ankG-binding AIS targeting motif, how do additional levels of subcellular specialization arise within the ankG-dependent AIS? Although the answer to this question remains unknown, some clues have come from recent experiments describing the existence of several different kinases at the AIS. For example, protein kinase CK2 was reported at the AIS and shown to phosphorylate several serine residues within the AIS targeting motif. Phosphorylation of these residues dramatically increased the affinity of $\mathrm{Na}^{+}$channels for ankG (Bréchet et al., 2008). It is easy to imagine a situation where regulation of CK2 activity could influence the density of $\mathrm{Na}^{+}$ channels at the AIS. CaMKII is also enriched at the AIS through interactions with $\beta \mathrm{IV}$ spectrin, and this in turn results in the phosphorylation of $\mathrm{Na}^{+}$channels (Fig. 1D) (Hund et al., 2010). Interestingly, the trafficking and insertion of axonal $\mathrm{K}_{\mathrm{V}} 1$ channels is controlled by an alternative set of kinases, including Cdk2 and Cdk5 (Vacher et al., 2011). These observations suggest that phosphorylation may play important roles in regulating AIS plasticity. Consistent with this idea, a very recent report identified an ankyrin-enriched AIS-like compartment in Drosophila mushroom-body $\gamma$-neurons whose length was modulated by Cdk5 activity (Trunova et al., 2011).

Although most of these results point to ankG as the key regulator of AIS assembly, function, and plasticity, important questions regarding ankG's function remain unanswered. In particular, how does ankG get recruited to the AIS? Determining how ankG's localization is regulated will no doubt provide key insights into the mechanisms of AIS plasticity.

\section{Neurological disorders and the AIS}

Both short- and long-term forms of plasticity at the AIS may play crucial roles in establishing and maintaining neuronal function. However, given the central role of the AIS in action potential initiation, it comes as no surprise that neurological disorders, in particular epilepsy, can arise as a result of AIS dysfunction. Numerous epilepsy-associated mutations have been found in key AIS ion channels, including $\mathrm{Na}^{+}, \mathrm{K}^{+}$, and $\mathrm{Ca}^{2+}$ channels as well as GABA receptor $\mathrm{Cl}^{-}$channels (Fig. $1 D$ ). The enrichment of epilepsy-related proteins at the AIS suggests that pathogenic pathways of many forms of epilepsy may converge at this subcellular compartment (for review, see Wimmer et al., 2010a). Recent work is beginning to unravel novel disease mechanisms anchored at the AIS (Schafer et al., 2009; Thomas et al., 2009; Wimmer et al., 2010b).

While the evidence linking AIS dysfunction to an epileptic phenotype is still largely correlative (Thomas et al., 2009; Liao et al., 2010; Wimmer et al., 2010a), detailed information about the cellular disease mechanism is available for a human epilepsy mutation in the gene coding for the accessory $\mathrm{Na}^{+}$channel subunit $\beta 1$ (SCN1B, Wimmer et al., 2010b). A C121W mutation in this SCN1B protein causes febrile seizures, a common childhood epilepsy (Wallace et al., 1998). Wimmer and colleagues created a heterozygous C121W knock in mouse model showing increased sensitivity to thermally triggered seizures, recapitulating febrile seizures seen in patients with the C121W mutation. Wild-type SCN1B subunits, as well as pore-forming sodium-channel $\alpha$ subunits, are found at the AIS membrane. However, the C121W mutation disrupts membrane localization of the mutant SCN1B subunit and consequently, SCN1B-mediated modulation of the $\alpha$ subunits. This "loss of function by absence" results in hyperpolarization of the activation voltage of $\mathrm{Na}^{+}$channels, a hyperpolarization of the action potential threshold and hyperexcitability of the affected neurons. Action potential initiation at the AIS is specifically affected while the somatic component of the action potential remains unchanged, suggesting that the disease mechanism is AIS specific. Intriguingly, an increase in temperature specifically and substantially enhances excitability of the AIS in the mutant neurons, indicating that increased temperature sensitivity may underlie development of febrile seizures.

Improving our understanding of cellular mechanisms of epileptogenesis in a range of syndromes will help establish whether AIS dysfunction as a cause for CNS disease is more common than currently thought and whether the AIS is indeed a "structure of convergence" whose functional properties are altered in a similar way by nonrelated epilepsy mutations as suggested previously (Wimmer et al., 2010a). Considering the large number of epilepsy-associated proteins enriched at the AIS, the concept of convergence holds great promise for novel epilepsy treatments because recent studies reveal a daunting variety of human genetic epilepsy mutations. Some epilepsy syndromes may share common, AIS-associated disease mechanisms, and recognizing these pathways may aid development of future anti-epileptic drugs.

\section{Conclusions}

These are exciting times for AIS biology. Although the structure itself has long been recognized, recent years have seen huge advances in our knowledge of fundamental AIS molecular anatomy, development, and function. It is now clear that these properties are not static; short- and long-term forms of plasticity at the AIS can produce significant alterations in action potential initiation and axodendritic patterning, and AIS-based alterations in ion channel function can be the cause of common epileptic disorders. Work in this fledgling field is picking up pace. In the coming years, a wide range of neuromodulatory molecules acting locally on action potential initiation may be uncovered, along with plastic changes that specifically affect the relative densities, subtypes, 
and distributions of AIS ion channels. We should soon have a clearer picture of the molecular mechanisms underlying AIS formation and maintenance, and should be able to directly relate changes in these mechanisms to AIS plasticity, ideally in vivo and under normal physiological conditions. Understanding diversity across neuronal types will be particularly crucial here. Finally, a wide range of neurological and psychological disorders have been associated with variation in genes coding for AIS components. We hope that a better knowledge of AIS development, maintenance, and plasticity will enable clearer understanding and improved treatment of these disorders in the near future.

\section{References}

Barnes AP, Polleux F (2009) Establishment of axon-dendrite polarity in developing neurons. Annu Rev Neurosci 32:347-381.

Bean BP (2007) The action potential in mammalian central neurons. Nat Rev Neurosci 8:451-465.

Bender KJ, Trussell LO (2009) Axon initial segment Ca2 + channels influence action potential generation and timing. Neuron 61:259-271.

Bender KJ, Ford CP, Trussell LO (2010) Dopaminergic modulation of axon initial segment calcium channels regulates action potential initiation. Neuron 68:500-511.

Bréchet A, Fache MP, Brachet A, Ferracci G, Baude A, Irondelle M, Pereira S, Leterrier C, Dargent B (2008) Protein kinase CK2 contributes to the organization of sodium channels in axonal membranes by regulating their interaction with ankyrin G. J Cell Biol 183:1101-1114.

Callewaert G, Eilers J, Konnerth A (1996) Axonal calcium entry during fast 'sodium' action potentials in rat cerebellar Purkinje neurones. J Physiol 495:641-647.

Chand AN, Grubb MS (2011) Activity-dependent plasticity in dopaminergic neurons of the olfactory bulb. Soc Neurosci Abstr 37:331.01.

Colbert CM, Pan E (2002) Ion channel properties underlying axonal action potential initiation in pyramidal neurons. Nat Neurosci 5:533-538.

Davis JQ, Bennett V (1994) Ankyrin binding activity shared by the neurofascin/L1/NrCAM family of nervous system cell adhesion molecules. J Biol Chem 269:27163-27166.

Evans MD, Grubb MS (2011) Mechanisms of activity-dependent plasticity at the axon initial segment. Soc Neurosci Abstr 37:874.04.

Fleidervish IA, Lasser-Ross N, Gutnick MJ, Ross WN (2010) Na+ imaging reveals little difference in action potential-evoked $\mathrm{Na}+$ influx between axon and soma. Nat Neurosci 13:852-860.

Fried SI, Lasker AC, Desai NJ, Eddington DK, Rizzo JF 3rd (2009) Axonal sodium-channel bands shape the response to electric stimulation in retinal ganglion cells. J Neurophysiol 101:1972-1987.

Garrido JJ, Giraud P, Carlier E, Fernandes F, Moussif A, Fache MP, Debanne D, Dargent B (2003) A targeting motif involved in sodium channel clustering at the axonal initial segment. Science 300:2091-2094.

Goldberg EM, Clark BD, Zagha E, Nahmani M, Erisir A, Rudy B (2008) K+ channels at the axon initial segment dampen near-threshold excitability of neocortical fast-spiking GABAergic interneurons. Neuron 58:387-400.

Grubb MS, Burrone J (2010) Activity-dependent relocation of the axon initial segment fine-tunes neuronal excitability. Nature 465:1070-1074.

Hedstrom KL, Xu X, Ogawa Y, Frischknecht R, Seidenbecher CI, Shrager P, Rasband MN (2007) Neurofascin assembles a specialized extracellular matrix at the axon initial segment. J Cell Biol 178:875-886.

Hedstrom KL, Ogawa Y, Rasband MN (2008) AnkyrinG is required for maintenance of the axon initial segment and neuronal polarity. J Cell Biol 183:635-640.

Hill AS, Nishino A, Nakajo K, Zhang G, Fineman JR, Selzer ME, Okamura Y, Cooper EC (2008) Ion channel clustering at the axon initial segment and node of Ranvier evolved sequentially in early chordates. PLoS Genet 4:e1000317.

Hu W, Tian C, Li T, Yang M, Hou H, Shu Y (2009) Distinct contributions of $\mathrm{Na}(\mathrm{v}) 1.6$ and $\mathrm{Na}(\mathrm{v}) 1.2$ in action potential initiation and backpropagation. Nat Neurosci 12:996-1002.

Hund TJ, Koval OM, Li J, Wright PJ, Qian L, Snyder JS, Gudmundsson H, Kline CF, Davidson NP, Cardona N, Rasband MN, Anderson ME, Mohler PJ (2010) A beta(IV)-spectrin/CaMKII signaling complex is essential for membrane excitability in mice. J Clin Invest 120:3508-3519.

Kole MH, Letzkus JJ, Stuart GJ (2007) Axon initial segment Kv1 channels control axonal action potential waveform and synaptic efficacy. Neuron 55:633-647.

Kole MH, Ilschner SU, Kampa BM, Williams SR, Ruben PC, Stuart GJ (2008) Action potential generation requires a high sodium channel density in the axon initial segment. Nat Neurosci 11:178-186.

Konishi M (2003) Coding of auditory space. Annu Rev Neurosci 26:31-55.

Kuba H, Ohmori H (2009) Roles of axonal sodium channels in precise auditory time coding at nucleus magnocellularis of the chick. J Physiol 587:87-100.

Kuba H, Ishii TM, Ohmori H (2006) Axonal site of spike initiation enhances auditory coincidence detection. Nature 444:1069-1072.

Kuba H, Oichi Y, Ohmori H (2010) Presynaptic activity regulates $\mathrm{Na}(+)$ channel distribution at the axon initial segment. Nature 465:1075-1078.

Lemaillet G, Walker B, Lambert S (2003) Identification of a conserved ankyrin-binding motif in the family of sodium channel alpha subunits. J Biol Chem 278:27333-27339.

Liao Y, Anttonen AK, Liukkonen E, Gaily E, Maljevic S, Schubert S, BellanKoch A, Petrou S, Ahonen VE, Lerche H, Lehesjoki AE (2010) SCN2A mutation associated with neonatal epilepsy, late-onset episodic ataxia, myoclonus, and pain. Neurology 75:1454-1458.

Lorincz A, Nusser Z (2008) Cell-type-dependent molecular composition of the axon initial segment. J Neurosci 28:14329-14340.

Lüscher C, Lipp P, Lüscher HR, Niggli E (1996) Control of action potential propagation by intracellular $\mathrm{Ca} 2+$ in cultured rat dorsal root ganglion cells. J Physiol 490:319-324.

Marder E, Prinz AA (2002) Modeling stability in neuron and network function: the role of activity in homeostasis. Bioessays 24:1145-1154.

Ogawa Y, Horresh I, Trimmer JS, Bredt DS, Peles E, Rasband MN (2008) Postsynaptic density-93 clusters Kv1 channels at axon initial segments independently of Caspr2. J Neurosci 28:5731-5739.

Palmer LM, Stuart GJ (2006) Site of action potential initiation in layer 5 pyramidal neurons. J Neurosci 26:1854-1863.

Pan Z, Kao T, Horvath Z, Lemos J, Sul J-Y, Cranstoun SD, Bennett V, Scherer SS, Cooper EC (2006) A common ankyrin-G-based mechanism retains KCNQ and Nav channels at electrically active domains of the axon. J Neurosci 26:2599-2613.

Perez-Reyes E (2003) Molecular physiology of low-voltage-activated t-type calcium channels. Physiol Rev 83:117-161.

Popovic MA, Foust AJ, McCormick DA, Zecevic D (2011) The spatiotemporal characteristics of action potential initiation in layer 5 pyramidal neurons: a voltage-imaging study. J Physiol 589:4167-4187.

Sánchez-Ponce D, DeFelipe J, Garrido JJ, Muñoz A (2011) In vitro maturation of the cisternal organelle in the hippocampal neuron's axon initial segment. Mol Cell Neurosci 48:104-116.

Schafer DP, Jha S, Liu F, Akella T, McCullough LD, Rasband MN (2009) Disruption of the axon initial segment cytoskeleton is a new mechanism for neuronal injury. J Neurosci 29:13242-13254.

Schiller J, Helmchen F, Sakmann B (1995) Spatial profile of dendritic calcium transients evoked by action potentials in rat neocortical pyramidal neurones. J Physiol 487:583-600.

Shu Y, Hasenstaub A, Duque A, Yu Y, McCormick DA (2006) Modulation of intracortical synaptic potentials by presynaptic somatic membrane potential. Nature 441:761-765.

Shu Y, Yu Y, Yang J, McCormick DA (2007a) Selective control of cortical axonal spikes by a slowly inactivating K+ current. Proc Natl Acad Sci U S A 104:11453-11458.

Shu Y, Duque A, Yu Y, Haider B, McCormick DA (2007b) Properties of action-potential initiation in neocortical pyramidal cells: evidence from whole cell axon recordings. J Neurophysiol 97:746-760.

Sobotzik JM, Sie JM, Politi C, Del Turco D, Bennett V, Deller T, Schultz C (2009) Ankyring is required to maintain axo-dendritic polarity in vivo. Proc Natl Acad Sci U S A 106:17564-17569.

Thomas EA, Hawkins RJ, Richards KL, Xu R, Gazina EV, Petrou S (2009) Heat opens axon initial segment sodium channels: a febrile seizure mechanism? Ann Neurol 66:219-226.

Trunova S, Baek B, Giniger E (2011) Cdk5 regulates the size of an axon initial segment-like compartment in mushroom body neurons of the Drosophila central brain. J Neurosci 31:10451-10462.

Tucci DL, Rubel EW (1985) Afferent influences on brain stem auditory nuclei of the chicken: effects of conductive and sensorineural hearing loss on n. magnocellularis. J Comp Neurol 238:371-381. 
Turrigiano GG, Nelson SB (2004) Homeostatic plasticity in the developing nervous system. Nat Rev Neurosci 5:97-107.

Vacher H, Yang JW, Cerda O, Autillo-Touati A, Dargent B, Trimmer IS (2011) Cdk-mediated phosphorylation of the Kvbeta2 auxiliary subunit regulates Kv1 channel axonal targeting. J Cell Biol 192:813-824.

Van Wart A, Matthews G (2006) Expression of sodium channels Nav1.2 and Nav1.6 during postnatal development of the retina. Neurosci Lett 403:315-317.

Wallace RH, Wang DW, Singh R, Scheffer IE, George AL Jr, Phillips HA, Saar K, Reis A, Johnson EW, Sutherland GR, Berkovic SF, Mulley JC (1998) Febrile seizures and generalized epilepsy associated with a mutation in the $\mathrm{Na}+$-channel betal subunit gene SCN1B. Nat Genet 19:366-370.

Wimmer VC, Reid CA, So EY, Berkovic SF, Petrou S (2010a) Axon initial segment dysfunction in epilepsy. J Physiol 588:1829-1840.

Wimmer VC, Reid CA, Mitchell S, Richards KL, Scaf BB, Leaw BT, Hill EL, Royeck M, Horstmann MT, Cromer BA, Davies PJ, Xu R, Lerche H,
Berkovic SF, Beck H, Petrou S (2010b) Axon initial segment dysfunction in a mouse model of genetic epilepsy with febrile seizures plus. J Clin Invest 120:2661-2671.

Yang Y, Ogawa Y, Hedstrom KL, Rasband MN (2007) \{beta\}IV spectrin is recruited to axon initial segments and nodes of Ranvier by ankyrinG. J Cell Biol 176:509-519.

Yu Y, Maureira C, Liu X, McCormick D (2010) P/Q and N channels control baseline and spike-triggered calcium levels in neocortical axons and synaptic boutons. J Neurosci 30:11858-11869.

Zhou D, Lambert S, Malen PL, Carpenter S, Boland LM, Bennett V (1998) AnkyrinG is required for clustering of voltage-gated $\mathrm{Na}$ channels at axon initial segments and for normal action potential firing. J Cell Biol 143:1295-1304.

Zhu J, Jiang M, Yang M, Hou H, Shu Y (2011) Membrane potentialdependent modulation of recurrent inhibition in rat neocortex. PLoS Biol 9:e1001032. 